\title{
Uso de escitalopram em paciente com depressão secundária
}

\author{
Fabiano Coelho Horimoto* \\ Danusa Céspedes Guizzo Ayache* \\ Juberty Antônio de Souza**
}

\section{INTRODUÇÃO}

As depressões secundárias são decorrentes de uma doença física ou do uso de algum fármaco, com relação de causalidade direta entre o evento e o surgimento da depressão. A associação com outras condições clínicas, segundo Botega et al. ${ }^{1}$, pode ocorrer sob diversas modalidades: reação de ajustamento com humor depressivo, depressão secundária, transtorno depressivo induzido por medicamentos, dentre outros. Como muitos sintomas decorrentes das condições clínicas ${ }^{2}$ se sobrepõem aos sintomas depressivos, a avaliação da presença de transtorno depressivo é mais complexa.

Devido a isso, Zigmond \& Snaith ${ }^{3}$, em 1983, desenvolveram a Escala Hospitalar de Depressão e Ansiedade (HAD). A versão em

\footnotetext{
Trabalho realizado no Departamento de Clínica Médica da Universidade Federal de Mato Grosso do Sul (UFMS), Campo Grande, MS.

* Professor de Psiquiatria do Departamento de Clínica Médica da UFMS, Campo Grande, MS.

** Professor Assistente de Psiquiatria do Departamento de Clínica Médica da UFMS, Campo Grande, MS. Mestre em Saúde Coletiva pela UFMS, Campo Grande, MS.
}

português foi validada por Botega et al. ${ }^{4}$, e, em cada uma das subescalas, pontuações superiores a 7 sugerem a presença de quadros depressivos e ansiosos 5 .

Doenças crônicas, como diabetes ${ }^{6}$ e hepatite $\mathrm{C}^{7}$, freqüentemente estão associadas com o surgimento de quadros psiquiátricos, principalmente depressão. Nos diabéticos, há uma maior prevalência de depressão ${ }^{8}$ quando comparados à população geral, e os pacientes com esta comorbidade possuem um controle menos eficaz de sua doença clínica e, conseqüentemente, uma morbimortalidade aumentada.

$\mathrm{Na}$ hepatite $\mathrm{C}$, além do caráter crônico e prognóstico variável, o tratamento com interferon-alfa ${ }^{1,9}$ é descrito na literatura como passível de causar depressão, além de outros efeitos colaterais, como febre, mal-estar e prostração, que limitam muito as atividades do paciente. Além disso, segundo Dwight et al. ${ }^{7}$, há relatos documentando a presença de sintomas como fadiga e depressão na infecção crônica pelo vírus da hepatite C (HCV), além de déficit cognitivo.

Este artigo tem como objetivo ilustrar as 
dificuldades no diagnóstico/tratamento da depressão na vigência de outras doenças, como diabetes e hepatite C. Estas podem desencadear quadros depressivos, causados tanto pela patologia de base quanto pela terapêutica, como no caso do interferon.

\section{APRESENTAÇÃO DO CASO}

Paciente de 50 anos, casado, gerente de empresa de transportes, atendido em dezembro de 2001, quando se encontrava hospitalizado devido a descompensação diabética, com glicemia de $486 \mathrm{mg} / \mathrm{dl}$. A interconsulta foi solicitada devido a "sintomas depressivos e apatia + confusão mental". O paciente apresentava diabetes tipo II e hepatite C, diagnosticados em 1996, fazendo uso de hipoglicemiantes orais e interferon-alfa (este a partir de novembro de 2000). Na avaliação inicial, apresentava-se com rebaixamento da consciência, com dificuldades em fornecer informações, sendo estas colhidas com sua esposa. Ela referiu que, há 6 meses, o paciente voltou a fazer uso abusivo de álcool, ingerindoo diariamente, após o trabalho, chegando em casa embriagado, além de tomar as medicações de forma irregular, com exceção do interferon. Referiu também que o paciente se encontrava desgostoso com seu rendimento no trabalho e se sentia muito ansioso.

Devido ao quadro de delirium, optamos por não introduzir psicotrópicos. Após 3 dias, houve melhora do estado clínico. Na reavaliação, queixava-se de humor deprimido há 1 ano, após iniciar o interferon. Antes, sentia-se mais disposto, trabalhava normalmente e não apresentava sintomas depressivos. Com a medicação, sentia desânimo, dificuldades para trabalhar, com redução da memória e da concentração. Além disso, nos dias em que aplicava o interferon, tinha febre, mal-estar e fadiga. Negou sintomas hipomaníacos/ maníacos previamente. Referiu ter um irmão com alcoolismo; desconhecia outros casos de doenças mentais na família.

Iniciamos com 25 mg de nortriptilina à noite; o paciente teve alta após 7 dias, com 75 mg do tricíclico e lorazepam $1 \mathrm{mg}$ à noite. No retorno, após 1 semana, a dose foi aumentada para 125 mg. Houve melhora no sono, porém o paciente ainda se mantinha deprimido.

No dia 24 de dezembro de 2001, familiares telefonaram, pois o paciente saiu de casa e voltou embriagado e hostil. Foi internado e, no dia seguinte, disse estar se sentindo culpado por tudo e que a depressão estava mais intensa. Referiu ter iniciado uso de álcool aos 20 anos, para diminuir a ansiedade, principalmente em situações sociais. Quando teve confirmados os diagnósticos de hepatite e diabetes, ficou muito abatido, sentindo-se deprimido por algumas semanas; não procurou tratamento, havendo resolução espontânea. De 1996 a 2000, não apresentou sintomas depressivos - estes se iniciaram após a introdução do interferon.

Aumentamos a nortriptilina até $175 \mathrm{mg} / \mathrm{dia}$ e, após 66 dias de uso do tricíclico, devido à pouca melhora observada, suspendemos a medicação, trocando-a por sertralina, com aumento da dose até $200 \mathrm{mg} /$ dia. Com 2 meses de tratamento, devido à baixa resposta, o antidepressivo foi suspenso. Em maio de 2002, o paciente foi avaliado por neurologista e, nos exames complementares, apresentou tomografia de crânio com discreta atrofia frontotemporal e exame funcional (cintilografia) com hipoperfusão em região frontal.

Indicamos a eletroconvulsoterapia (ECT) e, após três sessões, houve suspensão do procedimento devido ao aumento da pressão arterial (PA) na $3^{\text {a }}$ sessão (PA: 240 x 140 $\mathrm{mmHg}$ ). Em seguida, usamos citalopram (julho de 2002) até $60 \mathrm{mg} / \mathrm{dia}$, com pouca melhora do humor, volição e ansiedade. O paciente persistia com o déficit cognitivo que apresentava mesmo antes da ECT.

Em março de 2003, aplicamos a Escala Hospitalar de Depressão e Ansiedade (HAD) ${ }^{4}$, com pontuação de 15 para ansiedade e 15 para depressão, e trocamos o antidepressivo para escitalopram na dose de $15 \mathrm{mg} / \mathrm{dia}$. Após 1 semana, foi introduzida amitriptilina a $25 \mathrm{mg} /$ noite, pois o paciente sentia dificuldades em conciliar o sono. Após 2 meses de tratamento, houve melhora do humor e ânimo, e o paciente voltou a fazer atividades que não conseguia realizar (caminhadas, dieta), embora as alterações cognitivas persistissem. Na reavaliação com a HAD, após 60 dias, as pontuações foram de 8 para ansiedade e 9 para depressão.

Em março de 2004, novamente aplicamos o teste, com pontuação de 6 e 9, respectivamente. Após essa consulta, optamos por aumentar o escitalopram para $20 \mathrm{mg} / \mathrm{dia}$, devido à presença de sintomas residuais de depressão. Houve controle da glicemia, e o interferon era usado durante períodos menores.

O humor e o ânimo atingiram um nível considerado bom pelo paciente e familiares, fato que não "ocorria há vários anos", segundo descreve sua esposa. 


\section{DISCUSSÃO}

Além do quadro de depressão secundária, ressaltamos que a associação com outras comorbidades psiquiátricas, como transtornos ansiosos e alcoolismo ${ }^{2}$, é muito comum, dificultando a adesão ao tratamento e levando a uma maior possibilidade de recaídas.

As alterações cerebrais que o paciente já apresentava podem se correlacionar com a redução na volição e com a disfunção cognitiva. A própria infecção pelo HCV, segundo alguns autores $^{7}$, pode gerar déficit cognitivo.

Quanto ao tratamento, Goodnick \& Hernandez ${ }^{9}$ sugerem o uso de fluoxetina, sertralina e nortriptilina para pacientes com diabetes, embora ressaltem a possibilidade desta última levar a aumento da glicemia, pela especificidade noradrenérgica da droga, e a ganho de peso. Houve a troca de nortriptilina para sertralina em monoterapia, que tem sido descrita como eficaz em pacientes com depressão induzida por interferon-alfa ${ }^{10}$. Cantrell et al. ${ }^{11}$ postulam que doses mais altas devem ser utilizadas para se obter resposta, sugerindo uma maior eficácia à medida que há um aumento de doses até $150-200 \mathrm{mg}$ ao dia. O paciente em questão, mesmo usando a sertralina em tempo e dose adequados, não melhorou, passando a usar o citalopram após a interrupção da ECT, que também é bastante eficaz nesses casos ${ }^{12}$.

Muitos autores ${ }^{13,14}$ relatam uma boa eficácia com citalopram em pacientes com depressão induzida por interferon: Hauser et al. ${ }^{13}$ reportam uma melhora de 11 dos 13 pacientes tratados. Gleason et al. ${ }^{14}$, num estudo aberto, observaram melhora - redução de $50 \%$ ou mais nos escores na escala HAM-D após 8 semanas - em 13 de 15 pacientes do estudo (dose média $=26,67 \mathrm{mg} / \mathrm{dia}$ ). Devido ao insucesso terapêutico com o citalopram, trocamos o medicamento pelo seu isômero, o escitalopram, apesar de não encontrarmos nenhuma referência bibliográfica (Medline) de pacientes com diabetes e hepatite $C$ tratados com esta droga. Através das avaliações objetiva (escala $\mathrm{HAD}^{3,4}$ ) e clínica, houve redução da sintomatologia depressiva, levando a uma maior adesão aos outros tratamentos e, conseqüentemente, a uma melhora global na saúde do paciente.

Com a discussão das terapêuticas utilizadas, incluindo uma droga recém-lançada (escitalopram), esperamos contribuir com o avanço nos estudos sobre opções de tratamento em casos de depressão grave ou associada a outras patologias. Porém, por se tratar apenas de um relato de caso, necessitamos futuramente de novos estudos, com desenho metodológico adequado, que possam confirmar esses achados, para que 0 clínico disponha de novas opções de tratamento.

\section{REFERÊNCIAS BIBLIOGRÁFICAS}

1. Botega NJ, Furlanetto L, Fráguas Jr R. Depressão no paciente clínico. In: Botega NJ, org. Prática psiquiátrica no hospital geral: interconsulta e emergência. Porto Alegre: Artmed; 2002. p.203-22.

2. Furlanetto L. Diagnóstico. In: Fráguas Jr R, Figueiró JAB. Depressões em medicina interna e em outras condições médicas - depressões secundárias. São Paulo: Atheneu; 2001. p.11-20.

3. Zigmond AS, Snaith RP. The Hospital Anxiety and Depression Scale. Acta Psychiatr Scand 1983;67:36170.

4. Botega NJ, Pondé M, Silveira DC, et al. Escala Hospitalar de Ansiedade e Depressão (HAD) em pacientes epilépticos ambulatoriais. J Bras Med 1998;47(6):285-9.

5. Botega NJ, Dalgalarrondo P. Avaliação do paciente. In: Botega NJ, org. Prática psiquiátrica no hospital geral: interconsulta e emergência. Porto Alegre: Artmed; 2002. p.145-66.

6. Goodnick PJ, Henry JH, Buki VM. Treatment of depression in patients with diabetes mellitus. J Clin Psychiatry 1995;56:128-36.

7. Dwight MM, Kowdley KV, Russo JE, Ciechanowski PS, Larson AM, Katon WJ. Depression, fatigue and functional disability in patients with chronic hepatitis C. J Psychosom Res 2000;49:311-7.

8. Gavard JA, Lustman PJ, Clouse RE. Prevalence of depression in adults with diabetes: an epidemiological evaluation. Diabetes Care 1993;16(8):1167-78.

9. Goodnick PJ, Hernandez M. Treatment of depression in comorbid medical illness. Expert Opin Pharmacother 2000;1(7):1367-84.

10. Schramm TM, Lawford BR, Macdonald GA, Cooksley WG. Sertraline treatment of interferon-alpha-induced depressive disorder. Med J Aust 2000;173(7):359-61.

11. Cantrell R, Gillespie W, Altshuler L. Fluoxetine and sertraline dosages in major depression. Depress Anxiety 1999;9(2):78-82.

12. Meleiro AMAS, Almeida AM. Uso de eletroconvulsoterapia no hospital geral. In: Rigonatti SP, Rosa MA. Indicação e prática da eletroconvulsoterapia. São Paulo: Lemos Editorial; 2000. p.117-30.

13. Hauser P, Khosla J, Aurora H, Laurin J, Kling MA, Hill J, et al. A prospective study of the incidence and open-label treatment of interferon-induced major depressive disorder in patients with hepatitis C. Mol Psychiatry 2002;7(9):9427.

14. Gleason OC, Yates WR, Isbell MD, Philipsen MA. An open-label trial of citalopram for major depression in patients with hepatitis C. J Clin Psychiatry 2002;63(3):194-8.

\section{RESUMO}

As depressões secundárias freqüentemente remetem o psiquiatra a dificuldades maiores no tratamento do paciente, visto ser necessário uma 
abordagem mais ampla do caso para que a terapêutica alcance o efeito esperado sem piorar as doenças de base e sem interagir com outros medicamentos prescritos. Este artigo ilustra o caso de paciente com diabetes tipo II e hepatite $C$ que, após fazer uso do interferon, apresentou sintomas depressivos. Estes só melhoraram, após diversas intervenções terapêuticas, com o uso do escitalopram. Também são discutidas as dificuldades no diagnóstico e tratamento da depressão e comorbidades psiquiátricas e clínicas de evolução crônica.

Descritores: Depressão, diabetes, hepatite $C$, eletroconvulsoterapia, escitalopram, depressão secundária.

\section{ABSTRACT}

Treatment of secondary depression frequently involves a higher degree of difficulty. Such cases require a broader approach, so that the therapy may reach the expected effect without worsening the underlying diseases and without interacting with other prescribed drugs. This article describes the case of a patient with type II diabetes and hepatitis $C$ who presented depressive symptoms after using interferon. Several drugs were tested, but symptoms only improved with the use of escitalopran. The authors also discuss the difficulty faced in the diagnosis and treatment of depression and psychiatric co-morbidities associated with the chronic illnesses presented by the patient.

Keywords: Depression, diabetes, hepatitis C escitalopran, electroconvulsotherapy, secondary depression.
Title: Use of escitalopram in a patient with secondary depression

\section{RESUMEN}

Las depresiones secundarias frecuentemente remiten el psiquiatra a dificultades mayores en el tratamiento del paciente, una vez que es necesario un planteo más amplio del caso para que la terapéutica logre el efecto esperado sin empeorar las enfermedades de base y sin interactuar con otros medicamentos prescritos. Este artículo ilustra el caso de un paciente con diabetes de tipo II y hepatitis $C$ que, después de utilizar el interferón, presentó síntomas depresivos. Éstos solo mejoraron después de diversas intervenciones terapéuticas, con el uso del escitalopram. También se discuten las dificultades en el diagnóstico y tratamiento de la depresión y comorbilidades psiquiátricas y clínicas de evolución crónica.

Palabras clave: Depresión, diabetes, hepatitis $C$, electroconvulsoterapia, escitalopram, depresión secundaria.

Título: Uso de escitalopram en paciente con depresión secundaria

Correspondência:

Fabiano Coelho Horimoto

Rua Rio Grande do Sul, 1590 - Vila Célia

CEP 79020-011 - Campo Grande - MS

E-mail: rdayache@terra.com.br

Fone: (67) 3026-7721/7723, (67) 9221-5151

Fax: (67) 324-6747

Copyright (C) Revista de Psiquiatria do Rio Grande do Sul - SPRS 